\title{
A General Inequality for CR-Warped Products in Generalized Sasakian Space Form and Its Applications
}

\author{
Yanlin Li $\mathbb{D}^{1}{ }^{1}$ Akram Ali $\mathbb{D}^{2},{ }^{2}$ and Rifaqat $\mathrm{Ali}^{3}$ \\ ${ }^{1}$ Department of Mathematics, Hangzhou Normal University, Hangzhou 311121, China \\ ${ }^{2}$ Department of Mathematics, College of Science, King Khalid University, 61413 Abha, Saudi Arabia \\ ${ }^{3}$ Department of Mathematics, College of Science and Arts, Muhayil, King Khalid University, P.O. Box 9004, \\ 61413 Abha, Saudi Arabia
}

Correspondence should be addressed to Akram Ali; akramali133@gmail.com

Received 9 May 2021; Accepted 23 July 2021; Published 10 August 2021

Academic Editor: Shyamal Kumar Hui

Copyright (C) 2021 Yanlin Li et al. This is an open access article distributed under the Creative Commons Attribution License, which permits unrestricted use, distribution, and reproduction in any medium, provided the original work is properly cited.

In the present paper, by considering the Gauss equation in place of the Codazzi equation, we derive new optimal inequality for the second fundamental form of CR-warped product submanifolds into a generalized Sasakian space form. Moreover, the inequality generalizes some inequalities for various ambient space forms.

\section{Introduction}

The fundamental idea of warped product manifolds was first initiated in [1] with manifolds of negative curvature. Let $N_{1}$ and $N_{2}$ be two Riemannian manifolds endowed with Riemannian matrices $g_{1}$ and $g_{2}$, respectively, such that $f: N_{1}$ $\longrightarrow(0, \infty)$ is a positive smooth function on $N_{1}$. Then, the warped product $M=N_{1} \times{ }_{f} N_{2}$ is characterized as the product manifold $N_{1} \times N_{2}$ with the equipped metric $g=g_{1}+f^{2}$ $g_{2}$. In particular, if $f=$ constant, then $M$ turned to be a Riemannian product manifold; otherwise, $M$ is called a nontrivial warped product manifold. Let $M=N_{1} \times{ }_{f} N_{2}$ be a nontrivial warped product manifold. Then,

$$
\nabla_{X} Z=\nabla_{Z} X=(X \ln f) Z,
$$

for any vector fields $X, Y \in \Gamma\left(T N_{1}\right)$ and $Z \in \Gamma\left(T N_{2}\right)$. If we consider a local orthonormal frame $\left\{e_{1}, e_{2}, \cdots, e_{n}\right\}$ such that $\left\{e_{i}\right\}_{1 \leq i \leq n_{1}} \in N_{1}$ and $\left\{e_{j}\right\}_{n_{1}+1 \leq j \leq n} \in N_{2}$, we have

$$
\sum_{1 \leq i \leq n_{1} n_{1}+1 \leq j \leq n} K\left(e_{i} \wedge e_{j}\right)=\frac{n_{2} \Delta f}{f} .
$$

In [2], Chen established the inequality for the squared norm of the mean curvature and the warping function $f$ of a CR-warped product $N_{T} \times{ }_{f} N_{\perp}$, where $N_{\perp}$ is a totally real submanifold and $N_{T}$ is a holomorphic submanifold, isometrically immersed in a complex space form as follows.

Theorem 1 (see [2]). $N_{T}^{n_{1}} \times{ }_{f} N_{\perp}^{n_{2}}$ be a CR-warped product into a complex space form $\tilde{M}(4 c)$ with constant sectional curvature c. Then,

$$
\|h\|^{2} \geq 2 n_{2}\left\{\|\nabla \ln f\|^{2}+\Delta(\ln f)+2 n_{1} c\right\},
$$

where $\Delta$ is the Laplacian operator of $N_{T}$. Moreover, the equality holds if and only if $N_{T}$ is totally geodesic and $N_{\perp}$ is totally umbilical in $\tilde{M}(4 c)$.

Moreover, Theorem 1 is extended to CR-warped product submanifolds in a generalized Sasakian space form by using the same technique.

Theorem 2 (see [3]). Let $N_{T}^{n_{1}} \times{ }_{f} N_{\perp}^{n_{2}}$ be a contact CR-warped product submanifold of a generalized Sasakian space form $\tilde{M}\left(\lambda_{1}, \lambda_{2}, \lambda_{3}\right)$ such that the structure vector field $\xi$ is tangent to base manifold. Then, the following inequality is satisfied: 


$$
\|h\|^{2} \geq 2 n_{2}\left(\|\nabla(\ln f)\|^{2}-\Delta(\ln f)+1\right)+4 n_{1} n_{2}\left(\lambda_{1}+1\right)
$$

where $\Delta$ denotes the Laplace operator on $N_{T}^{n_{1}}$. The equality holds if and only if $N_{T}^{n_{1}}$ is a totally geodesic submanifold of $\tilde{M}\left(\lambda_{1}, \lambda_{2}, \lambda_{3}\right)$; in this case, $N_{T}^{n_{1}}$ is a generalized Sasakian space form of $\left(\lambda_{1}+3 \lambda_{3}\right)$.

Furthermore, Mustafa et al. [4] recalled some fundamental problems of CR-warped products in Kenmotsu space forms as to simple relationships between the second fundamental form and the main intrinsic invariants by using the Gauss equation. In [5-7], some sharp inequalities are established for the sectional curvature of warped product pointwise semislant submanifolds in various space forms such as a Sasakian space form, a cosymplectic space form, a Kenmotsu space form, and a complex space form in terms of the Laplacian and the squared norm of a warping function with pointwise slant immersions. Afterward, several geometers $[1,2,4,8-18]$ obtained similar inequalities for different types of warped products in different kinds of structures.

Al-Ghefari et al. [3] proved the existence of CR-warped product submanifolds of type $N_{T} \times{ }_{f} N_{\perp}$ in trans-Sasakian manifolds. They obtained an inequality for the second fundamental form with constant sectional curvature in terms of a warping function. Moreover, the nonexistence of CRwarped products of the form $N_{\perp} \times{ }_{f} N_{T}$ in a generalized Sasakian space form was proved in [19].

In this paper, we shall establish a Chen-type inequality for CR-warped product submanifolds in a generalized Sasakian space form by considering the nontrivial case $N_{T} \times_{f}$ $N_{\perp}$. We also find some applications of the inequality in the compact Riemannian manifold by using integration theory on manifolds. Our future work then is combining the work done in this paper with the techniques of singularity theory presented in [20-23] to explore new results on manifolds.

\section{Preliminaries}

An almost contact metric manifold $(\tilde{M}, g, \varphi, \eta, \xi)$ is an odddimensional manifold $\tilde{M}$, endowed with a field $\varphi$ of an endomorphism on the tangent space, the Reeb vector field $\xi$, a 1form $\eta$ and admits Riemannian metric $g$ satisfying

$$
\begin{aligned}
\varphi^{2} & =-I+\eta \otimes \xi, \quad \eta(\xi)=1, \\
g(\varphi U, \varphi V) & =g(U, V)-\eta(X) \eta(Y), \\
\eta(U) & =g(U, \xi),
\end{aligned}
$$

for any $U, V \in \Gamma(T \tilde{M})$. An almost contact metric manifold $(\tilde{M}, g, \varphi, \eta, \xi)$ is said to be trans-Sasakian manifold (cf. $[12,13])$ if

$$
\left(\nabla^{\sim}{ }_{U} \varphi\right) V=\alpha(g(U, V) \xi-\eta(U) V)-\beta(g(\varphi U, V)-\eta(V) \varphi U),
$$

for any $U, V \in \Gamma(T \tilde{M})$, where $\tilde{\nabla}$ is the Riemannian connection on $(\tilde{M}, g)$. If we replace $U=\xi$ and $V=\xi$ in (8), we find that $\left(\nabla^{\sim}{ }_{\xi} \varphi\right) \xi=0$, which implies that $\nabla^{\sim}{ }_{\xi} \xi=0$. For a trans-Sasakian manifold, (8) implies

$$
\nabla_{X}^{\sim} \xi=-\alpha \varphi X+\beta(X-\eta(X) \xi)
$$

Remark 3. We classify a trans-Sasakian manifold in the following way:

(a) If $\alpha=0$ and $\beta=0$ in (8), a trans-Sasakian manifold becomes a cosymplectic manifold [7]

(b) If $\alpha=1$ and $\beta=0$ in (8), it is a Sasakian manifold [5]

(c) If $\alpha=0$ and $\beta=1$ in (8), it is a Kenmotsu manifold [6]

(d) $\alpha$-Sasakian manifold and $\beta$-Kenmotsu manifold can be derived from the tans-Sasakian manifold when $\beta=0$ and $\alpha=0$ in (8), respectively

Given an almost contact metric manifold $(\tilde{M}, \varphi, \eta, \xi)$, it is said to be a generalized Sasakian space form $\tilde{M}\left(\lambda_{1}, \lambda_{2}, \lambda_{3}\right)$ if there exist three functions $\lambda_{1}, \lambda_{2}$, and $\lambda_{3}$ on $\tilde{M}$ such that the curvature tensor $\tilde{R}$ is

$$
\begin{aligned}
\tilde{R}(X, Y, Z, W)= & \lambda_{1}(g(X, W) g(Y, Z)-g(X, Z) g(Y, W) \\
& +\lambda_{2}(g(X, \varphi Z) g(\varphi Y, W)-g(Y, \varphi Z) g(\varphi X, W) \\
& +2 g(X, \varphi Y) g(\varphi Z, W))+\lambda_{3}(\eta(X) \eta(Z) g(Y, W) \\
& -\eta(Y) \eta(Z) g(X, W)+g(X, Z) \eta(Y) \eta(W) \\
& -g(Y, Z) \eta(X) \eta(W))
\end{aligned}
$$

for any $X, Y, Z, W \in \Gamma(T \tilde{M})[24]$.

Remark 4. The characteristics are as follows:

(a) If $\lambda_{1}=c+3 / 4$ and $\lambda_{2}=\lambda_{3}=(c-1) / 4$, then $\tilde{M}$ is a Sasakian space form [25]

(b) If $\lambda_{1}=(c-3) / 4$ and $\lambda_{2}=\lambda_{3}=(c+1) / 4$, then $\tilde{M}$ is a Kenmotsu space form [6]

(c) If $\lambda_{1}=\lambda_{2}=\lambda_{3}=c / 4$, then $\tilde{M}$ is a cosymplectic space form [26]

Let $\nabla$ and $\nabla^{\perp}$ be the induced Riemannian connections on the tangent bundle $T M$ and the normal bundle $T^{\perp} M$ of a submanifold $M$ of an almost contact metric manifold $(\tilde{M}, \varphi, \eta, \xi)$ with the induced metric $g$. Then, the Gauss and Weingarten formulas are given by

$$
\text { (i) } \nabla^{\sim}{ }_{U} V=\nabla_{U} V+h(U, V),(\text { ii }) \nabla^{\sim}{ }_{U} N=-A_{N} U+\nabla_{U}^{\perp} N \text {, }
$$

for $U, V \in \Gamma(T M)$ and $N \in \Gamma\left(T^{\perp} M\right)$, where $h$ and $A_{N}$ are the second fundamental form and the shape operator on 
$M$. We have the relation:

$$
g(h(U, V), N)=g\left(A_{N} U, V\right)
$$

for $U, V \in \Gamma(T M)$ and $N \in \Gamma\left(T^{\perp} M\right)$. For any tangent vector $U \in \Gamma(T M)$ and normal vector $N \in \Gamma\left(T^{\perp} M\right)$, we have

$$
\begin{aligned}
& \text { (i) } \varphi U=T U+F U, \\
& \text { (ii) } \varphi N=t N+f N,
\end{aligned}
$$

where $T U(t N)$ and $F U(f N)$ are tangential and normal components of $\varphi U(\varphi N)$, respectively. If $T$ is identically zero, then a submanifold $M$ is called a totally real submanifold. The Gauss equation with curvature tensors $\tilde{R}$ and $R$ on $\tilde{M}$ and $M$, respectively, is defined by

$$
\begin{aligned}
\tilde{R}(U, V, Z, W)= & R(U, V, Z, W)+g(h(U, Z), h(V, W)) \\
& -g(h(U, W), h(V, Z))
\end{aligned}
$$

for any $U, V, Z, W \in \Gamma(T M)$. The mean curvature vector $H$ for a local frame $\left\{e_{1}, e_{2}, \cdots, e_{n}\right\}$ of the tangent space $T$ $M$ on $M$ is defined by

$$
\begin{aligned}
& \text { (i) } H=\frac{1}{n} \operatorname{trace}(h)=\frac{1}{n} \sum_{i=1}^{n} h\left(e_{i}, e_{i}\right), \\
& \text { (ii) }\|T\|^{2}=\sum_{i, j=1}^{n} g^{2}\left(\varphi e_{i}, e_{j}\right) .
\end{aligned}
$$

The scalar curvature $\tau$ for a Riemannian submanifold $M$ is given by

$$
\tau=\sum_{1 \leq i=j \leq n} K\left(e_{i} \wedge e_{j}\right)
$$

where $K\left(e_{i} \wedge e_{j}\right)$ is the sectional curvature of section plane and spanned by $e_{i}$ and $e_{j}$. Let $G_{r}$ be an $r$-plane section on $T M$ and let $\left\{e_{1}, e_{2}, \cdots, e_{r}\right\}$ be a orthonormal basis of $G_{r}$. Then, the scalar curvature $\tau\left(G_{r}\right)$ of $G_{r}$ is given by

$$
\tau\left(G_{r}\right)=\sum_{1 \leq i=j \leq r} K\left(e_{i} \wedge e_{j}\right) .
$$

Similarly, we classify a Riemannian submanifold $M$ said to be totally umbilical and totally geodesic if $h(U, V)$ $=g(U, V) H$ and $h(U, V)=0$, respectively, for any $U, V$ $\in \Gamma(T M)$.

Furthermore, if $H=0$, then $M$ is minimal in $(\tilde{M}, \varphi, \eta, \xi)$. If $\varphi$ preserves any tangent space of $M$ tangent to the structure vector field $\xi$, i.e., $\varphi\left(T_{p} M\right) \subseteq T_{p} M$, for each $p \in M$; then, $M$ is called an invariant submanifold. Similarly, $M$ is called an anti-invariant submanifold tangent to the Reeb vector field $\xi$ if $\varphi\left(T_{p} M\right) \subseteq T^{\perp} M$, for each $p \in M$. To generalize these definitions, we give the following definition.
Definition 5. A submanifold $M$ including the structure vector field $\xi$ of an almost contact metric manifold $(\tilde{M}, \varphi, \eta, \xi)$ is characterized to be a contact CR-submanifold if the pair of orthogonal distributions $\mathscr{D}$ and $\mathscr{D}^{\perp}$ exists such that

(i) $T M=\mathscr{D} \oplus \mathscr{D}^{\perp} \oplus\langle\xi\rangle$, where $\langle\xi\rangle$ is 1-dimensional distribution spanned by $\xi$

(ii) the distribution $\mathscr{D}$ is invariant, i.e., $\varphi(\mathscr{D}) \subseteq \mathscr{D}$

(iii) the distribution $\mathscr{D}^{\perp}$ is anti-invariant, i.e., $\varphi \mathscr{D}^{\perp} \subseteq$ $\left(T^{\perp} M\right)$

If the dimensions of invariant distribution $\mathscr{D}$ and antiinvariant distribution $\mathscr{D}^{\perp}$ of a contact CR-submanifold of $(\tilde{M}, \varphi, \eta, \xi)$ are $d_{1}$ and $d_{2}$, respectively, such that $d_{2}=0$, then $M$ is invariant and anti-invariant if $d_{1}=0$. It is called a proper contact CR-submanifold if neither $d_{1}=0$ nor $d_{2}=0$. The normal bundle $T^{\perp} M$ of a contact CR-submanifold with an invariant subspace $\mu$ under $\varphi$ can be decomposed as

$$
T^{\perp} M=\varphi \mathscr{D}^{\perp} \oplus \mu .
$$

$M$ is a compact orientable Riemannian submanifold without boundary. Thus, we have

$$
\int_{M} \Delta f d V=0
$$

where $d V$ is the volume element of $M$ [27].

\section{Main Inequalities of CR-Warped Products}

We are mentioning that in the following study, we shall consider the structure field $\xi$ tangent to the base manifold of warped product manifold. In this main section, we classify the contact CR-warped product submanifolds in a transSasakian manifold.

Lemma 6. Let $M=N_{T} \times{ }_{f} N_{\perp}$ be a CR-warped product submanifold in a trans-Sasakian manifold. Then,

$$
\begin{gathered}
g(h(\varphi X, Y), \varphi Z)=g(h(X, Y), \varphi Z)=0, \\
g(h(X, X), \beta)=-g(h(\varphi X, \varphi X), \beta),
\end{gathered}
$$

for $X, Y \in \Gamma\left(T N_{T}\right), Z, W \in \Gamma\left(T N_{\perp}\right)$, and $\beta \in \Gamma(\mu)$.

Proof. From (11)(i), (8), and (5), we obtain

$$
g(h(\varphi X, Y), \varphi Z)=g\left(\bar{\nabla}_{Y} \varphi X, \varphi Z\right)=g\left(\bar{\nabla}_{Y} X, Z\right)-\eta\left(\bar{\nabla}_{Y} X\right) \eta(Z)
$$

Since $N_{T}$ is totally geodesic in $M$ with $\xi \in \Gamma\left(T N_{T}\right)$, (9) implies the results.

Lemma 7. Let $\ell: M=N_{T} \times{ }_{f} N_{\perp} \longrightarrow(\tilde{M}, \varphi, \eta, \xi)$ be an isometric immersion from an $n$-dimensional contact $C R$ - 
warped product submanifold into a trans-Sasakian manifold $(\tilde{M}, \varphi, \eta, \xi)$ such that $N_{T}$ is invariant submanifold of dimension $n_{1}=2 d_{1}+1$ tangent to $\xi$. Then, $N_{T}$ is always $\ell$-minimal submanifold of $\tilde{M}$.

Proof. We skip the proof of the above lemma due to the similar proof of Theorem 4.2 in [4].

By helping the above lemma, the following result can be obtained as follows.

Proposition 8. Assume that $\ell: M=N_{T} \times{ }_{f} N_{\perp} \longrightarrow \tilde{M}$ is an isometric immersion of an $n$-dimensional contact $C R$ warped product submanifold $M$ into a trans-Sasakian manifold $\tilde{M}$. Thus,

(i) the squared norm of the second fundamental form of $M$ is satisfied:

$$
\|h\|^{2} \geq 2\left(n_{2}\|\nabla \ln f\|^{2}+\tilde{\tau}(T M)-\tilde{\tau}\left(T N_{T}\right)-\tilde{\tau}\left(T N_{\perp}\right)-n_{2} \Delta(\ln f)\right),
$$

where $n_{2}$ is the dimension of anti-invariant submanifold $N_{\perp}$ and $\Delta$ is the Laplacian operator of $N_{T}$

(ii) the equality holds in (22) if and only if $N_{T}$ is totally geodesic and $N_{\perp}$ is totally umbilical in $\tilde{M}$. Moreover, $M$ is minimal submanifold of $\tilde{M}$

Proof. It can be easily proven as the proof of Theorem 4.4 in [4] if we consider a Riemannian submanifold as a CR-warped product submanifold, and the base manifold is a transSasakian manifold instead of a Kenmotsu manifold.

Now, we prove our main theorem using Proposition 8 for a generalized Sasakian space form.

Theorem 9. Let $\ell: M=N_{T} \times{ }_{f} N_{\perp} \longrightarrow \tilde{M}\left(\lambda_{1}, \lambda_{2}, \lambda_{3}\right)$ be an isometric immersion from an $n$-dimensional contact $C R$ warped product submanifold of a generalized Sasakian space form $\tilde{M}\left(\lambda_{1}, \lambda_{2}, \lambda_{3}\right)$. Then, the second fundamental form is given by

$$
\|h\|^{2} \geq 2 n_{2}\left\{\|\nabla \ln f\|^{2}+\lambda_{1} n_{1}+\frac{3}{2} \lambda_{2}-\lambda_{3}-\Delta(\ln f)\right\}
$$

where $n_{1}=\operatorname{dim} N_{T}, n_{2}=\operatorname{dim} N_{\perp}$, and $\Delta$ is the Laplacian operator on $N_{T}$. The equality holds in (23) if and only if $N_{T}$ and $N_{\perp}$ are totally geodesic and totally umbilical submanifolds in $\tilde{M}\left(\lambda_{1}, \lambda_{2}, \lambda_{3}\right)$, respectively, and hence, $M$ is a minimal submanifold of $\tilde{M}\left(\lambda_{1}, \lambda_{2}, \lambda_{3}\right)$.
Proof. Substituting $X=W=e_{i}$ and $Y=Z=e_{j}$ in (10), we get

$$
\begin{aligned}
\tilde{R}\left(e_{i}, e_{j}, e_{j}, e_{i}\right)= & \lambda_{1}\left\{g\left(e_{i}, e_{i}\right) g\left(e_{j}, e_{j}\right)-g\left(e_{i}, e_{j}\right) g\left(e_{i}, e_{j}\right)\right\} \\
& +\lambda_{2}\left\{g\left(e_{i}, \varphi e_{j}\right) g\left(\varphi e_{j}, e_{i}\right)-g\left(e_{i}, \varphi e_{i}\right) g\left(e_{j}, \varphi e_{j}\right)\right. \\
& \left.+2 g^{2}\left(\varphi e_{j}, e_{i}\right)\right\}+\lambda_{3}\left\{g\left(e_{i}, e_{i}\right) \eta\left(e_{j}\right) \eta\left(e_{j}\right)\right. \\
& -g\left(e_{i}, e_{j}\right) \eta\left(e_{i}\right) \eta\left(e_{i}\right)+g\left(e_{j}, e_{j}\right) \eta\left(e_{i}\right) \eta\left(e_{i}\right) \\
& \left.\cdot g\left(e_{j}, e_{i}\right)\left(e_{i}\right) \eta\left(e_{j}\right)\right\} .
\end{aligned}
$$

Summing up along the orthonormal vector fields of $M$, it can be derived from the above as

$$
2 \tilde{\tau}(T M)=\lambda_{1} n(n-1)+3 \lambda_{2} \sum_{1 \leq i=j \leq n} g^{2}\left(\varphi e_{i}, e_{j}\right)-2 \lambda_{3}(n-1) .
$$

As for an $n$-dimensional CR-warped product submanifold tangent $\xi$, one can derive $\|T\|^{2}=n-1$ from (15)(ii); we obtain

$$
2 \tilde{\tau}(T M)=\lambda_{1} n(n-1)+3(n-1) \lambda_{2}-2 \lambda_{3}(n-1) .
$$
have

On the other hand, by helping the frame field of $T N_{\perp}$, we

$$
2 \tilde{\tau}\left(T N_{\perp}\right)=\lambda_{1} n_{2}\left(n_{2}-1\right) .
$$

Similarly, we considered that $\xi$ is tangent to invariant submanifold $N_{T}$. Then, using the frame vector fields of $T$ $N_{T}$, we get from (24)

$$
2 \tilde{\tau}\left(T N_{T}\right)=\lambda_{1} n_{1}\left(n_{1}-1\right)+3 \lambda_{2}\left(n_{1}-1\right)-2 \lambda_{3}\left(n_{1}-1\right) .
$$

Therefore, using (26), (27), and (28) in Proposition 8, we get the required result. The equality case follows from Proposition 8. Thus, the proof is completed.

\section{Geometric Applications}

Remark 10. Consider $\lambda_{1}=(c-3) / 4$ and $\lambda_{2}=\lambda_{3}=(c+1) / 4$ in Theorem 9. It is the generalization of Theorem 4.6 in [4] for the result of contact CR-warped products in Kenmotsu space forms.

Remark 11. If we put $\lambda_{1}=(c+3) / 4$ and $\lambda_{2}=\lambda_{3}=(c-1) / 4$ in Theorem 9, then it generalizes Corollary 4.6 in [5].

Remark 12. If $\lambda_{1}=\lambda_{2}=\lambda_{3}=c / 4$ in Theorem 9, then Theorem 9 coincides with Theorem 1.2 in [26].

Corollary 13. Let $\ln f$ be a harmonic function on $N_{T}$. Then there does not exist any CR-warped product submanifold $N_{T}$ $\times{ }_{f} N_{\perp}$ into a generalized Sasakian space form $\tilde{M}\left(\lambda_{1}, \lambda_{2}, \lambda_{3}\right)$ with $c \leq-\lambda_{1}$. 
Corollary 14. Assume that $\ln f$ is a nonnegative eigenfunction on $N_{T}$ with the corresponding nonzero positive eigenvalue. Then, there does not exist any CR-warped product submanifold $N_{T} \times{ }_{f} N_{\perp}$ into a generalized Sasakian space form $\tilde{M}\left(\lambda_{1}, \lambda_{2}, \lambda_{3}\right)$ with $c \leq-\lambda_{1}$.

Theorem 15. Let $M=N_{T} \times{ }_{f} N_{\perp}$ be a compact orientated CR-warped product into a generalized Sasakian space form $\tilde{M}\left(\lambda_{1}, \lambda_{2}, \lambda_{3}\right)$. Then, $M$ is a simply Riemannian product if

$$
\|h\|^{2} \geq 2 \lambda_{1} n_{1} n_{2}+3 \lambda_{2} n_{2}-2 \lambda_{3} n_{2}
$$

where $n_{1}=\operatorname{dim} N_{T}$ and $n_{2}=\operatorname{dim} N_{\perp}$.

Proof. From Theorem 9, we get

$\|h\|^{2} \geq 2 \lambda_{1} n_{1} n_{2}+3 \lambda_{2} n_{2}-2 \lambda_{3} n_{3}-n_{2} \Delta(\ln f)+n_{2}\|\nabla \ln f\|^{2}$,

$n_{2}\|\nabla \ln f\|^{2}+2 \lambda_{1} n_{1} n_{2}+3 \lambda_{2} n_{2}-2 \lambda_{3} n_{3}-\|h\|^{2} \leq n_{2} \Delta(\ln f)$.

We obtain

$$
\begin{aligned}
& \int_{N_{T} \times q}\left(2 \lambda_{1} n_{1} n_{2}+3 \lambda_{2} n_{2}-2 \lambda_{3} n_{2}+n_{2}\|\nabla \ln f\|^{2}-\|h\|^{2}\right) \mathrm{d} V \\
& \quad \leq n_{2} \int_{N_{T} \times q} \Delta(\ln f) \mathrm{d} V=0 .
\end{aligned}
$$

Now, if

$$
\|h\|^{2} \geq 2 \lambda_{1} n_{1} n_{2}+3 \lambda_{2} n_{2}-2 \lambda_{3} n_{2}
$$

Then, from (31), we find

$$
\int_{N_{T} \times q}\left(\|\nabla \ln f\|^{2}\right) d V \leq 0
$$

which is impossible for a positive integral function, and hence, $\nabla \ln f=0$, i.e., $f$ is a constant function on $N_{T}$. Thus, by the definition of a warped product manifold, $M$ is trivial. The converse part is straightforward.

Corollary 16. Assume that $M=N_{T} \times{ }_{f} N_{\perp}$ is a CR-warped product submanifold in a generalized Sasakian space form $\tilde{M}\left(\lambda_{1}, \lambda_{2}, \lambda_{3}\right)$. Let $N_{T}$ be a compact invariant submanifold and $\gamma$ be nonzero eigenvalue of the Laplacian on $N_{T}$. Then,

$$
\begin{aligned}
& \int_{N_{T} \times q}\|h\|^{2} d V_{T} \geq\left(2 \lambda_{1} n_{1} n_{2}+3 \lambda_{2} n_{2}-2 \lambda_{3} n_{2}\right) \operatorname{Vol}\left(N_{T}\right) \\
& \quad+2 n_{2} \gamma \int_{N_{T} \times q}(\ln f)^{2} d V_{T} .
\end{aligned}
$$

Proof. From the minimum principle property, we obtain

$$
\int_{N_{T}}\|\nabla \ln f\|^{2} d V_{T} \geq \gamma \int_{N_{T}}(\ln f)^{2} d V_{T}
$$

From (23) and (35), we get the required result (34).

\section{Data Availability}

There is no data used for this manuscript.

\section{Conflicts of Interest}

The authors declare no competing interest.

\section{Authors' Contributions}

All authors have equal contribution and finalized.

\section{Acknowledgments}

The authors extend their appreciation to the deanship of scientific research at King Khalid University for funding this work through a research group program under grant number R.G.P.2/71/41.

\section{References}

[1] R. L. Bishop and B. O’Neill, "Manifolds of negative curvature," Transactions of the American Mathematical Society, vol. 145, pp. 1-9, 1969.

[2] B. Y. Chen, "Another general inequality for CR-warped products in complex space forms," Hokkaido Mathematical Journal, vol. 32, pp. 415-444, 2003.

[3] R. Al-Ghefari, F. R. Al-Solamy, and M. H. Shahid, "Contact CR-warped product submanifolds in generalized Sasakian space forms," Balkan Journal of Geometry and its Applications, vol. 11, pp. 1-9, 2006.

[4] A. Mustafa, A. De, and S. Uddin, "Characterization of warped product submanifolds in Kenmotsu manifolds," Balkan Journal of Geometry and its Applications, vol. 20, no. 1, pp. 8697, 2015.

[5] A. Akram and L. I. Pişcoran, "Geometric classification of warped products isometrically immersed into Sasakian space forms," Mathematische Nachrichten, vol. 292, no. 2, pp. 234$251,2019$.

[6] A. Ali and P. Laurian-Ioan, "Geometry of warped product immersions of Kenmotsu space forms and its applications to slant immersions," Journal of Geometry and Physics, vol. 114, pp. 276-290, 2017.

[7] A. Ali and C. Ozel, "Geometry of warped product pointwise semi-slant submanifolds of cosymplectic manifolds and its applications," International Journal of Geometric Methods in Modern Physics, vol. 14, no. 3, article 1750042, 2017.

[8] I. al-Dayel and M. Ali Khan, "Ricci curvature of contact CRwarped product submanifolds in generalized Sasakian space forms admitting nearly Sasakian structure," AIMS Mathematics, vol. 6, no. 3, pp. 2132-2151, 2021.

[9] N. Alluhaibi and M. A. Khan, "Warped product pointwise semi slant submanifolds of Sasakian space forms and their 
applications," Advances in Mathematical Physics, vol. 2020, Article ID 5654876, 13 pages, 2020.

[10] D. E. Blair and D. K. Showers, "Almost contact manifolds with killing structures tensors II," Journal of Differential Geometry, vol. 9, pp. 577-582, 1974.

[11] B. Y. Chen, "Geometry of warped product submanifolds: a survey," Journal of Advanced Mathematical Studies, vol. 6, no. 2, pp. 1-43, 2013.

[12] S. Deshmukh, "Trans-Sasakian manifolds homothetic to Sasakian manifolds," Mediterranean Journal of Mathematics, vol. 13, no. 5, pp. 2951-2958, 2016.

[13] S. Deshmukh and F. al-Solamy, "A note on compact transSasakian manifolds," Mediterranean Journal of Mathematics, vol. 13, no. 4, pp. 2099-2104, 2016.

[14] S. Dirik, "On some geometric properties of CR-submanifolds of a Sasakian manifold," Journal of Geometry and Physics, vol. 154, 2020.

[15] M. A. Khan, A. H. Alkhaldi, L. S. Alqahtani, and K. Khan, "Contact CR-warped product submanifolds of generalized Sasakian space forms admitting Ricci soliton," International Journal of Geometric Methods in Modern Physics, vol. 17, no. 1, article 2050009, p. 12, 2020.

[16] P. Mandal, T. Pal, and S. K. Hui, "Ricci curvature on warped product submanifolds of Sasakian space forms," Filomat, vol. 34, no. 12, pp. 3917-3930, 2020.

[17] A. Mustafa, S. Uddin, V. Khan, and B. R. Wong, "Contact CRwarped product submanifolds of nearly trans-Sasakian manifolds," Taiwanese Journal of Mathematics, vol. 17, no. 4, pp. 1473-1486, 2013.

[18] M. I. Munteanu, "Warped product contact CR-submanifolds of Sasakian space forms," Publicationes Mathematicae Debrecen, vol. 66, pp. 75-120, 2005.

[19] S. Sular and C. Ozgur, "Contact CR-warped product submanifolds in generalized Sasakian space forms," Turkish Journal of Mathematics, vol. 36, pp. 485-497, 2012.

[20] Y. Li, S. Liu, and Z. Wang, "Tangent developables and Darboux developables of framed curves," Topology and its Applications, p. 103639, 2020.

[21] Y. Li and Z. Wang, "Lightlike tangent developables in de Sitter 3-space," Journal of Geometry and Physics, vol. 164, pp. 1-11, 2021.

[22] Y. Li, Z. Wang, and T. Zhao, "Some results for the two-sided quaternionic Gabor fourier transform and quaternionic Gabor frame operator," Advances in Applied Clifford Algebras, vol. 31, no. 1, pp. 1-19, 2021.

[23] Y. Li, Y. Zhu, and Q. Sun, "Singularities and dualities of pedal curves in pseudo-hyperbolic and de Sitter space," International Journal of Geometric Methods in Modern Physics, vol. 18, pp. 1-31, 2021.

[24] P. Alegre, D. E. Blair, and A. Carriazo, "Generalized Sasakianspace-forms," Israel Journal of Mathematics, vol. 141, no. 1, pp. 157-183, 2004.

[25] M. Atceken, "Contact CR-warped product submanifolds in Sasakian space forms," Hacettepe Journal of Mathematics and Statistics, vol. 44, pp. 23-32, 2015.

[26] S. Uddin and L. S. Alqahtani, "Chen type inequality for warped product immersions in cosymplectic space forms," Journal of Nonlinear Sciences and Applications, vol. 9, no. 5, pp. 29142921, 2016.

[27] K. Yano and M. Kon, Structures on Manifolds, World Scientific, 1984. 